\title{
NA65/DsTau: Study of tau-neutrino production at the CERN SPS
}

\section{Tomoko Ariga* for the DsTau Collaboration}

Kyushu University

E-mail: tomoko.ariga@cern.ch

DsTau is an experiment at the CERN SPS, approved by CERN in June 2019 as NA65, to study $v_{\tau}$ production aiming at providing important data for future $v_{\tau}$ studies. A precise measurement of the $v_{\tau}$ cross section would enable a search for new physics effects in $v_{\tau}$ CC interactions. It also has practical importance for the next generation experiments for neutrino oscillation studies and astrophysical $v_{\tau}$ observations. The practical way of producing a $v_{\tau}$ beam is by the sequential decay of $D_{s}$ mesons produced in high-energy proton interactions. However, there is no experimental measurement of the $D_{s}$ differential production cross section in fixed target experiments using proton beams, which leads to a large systematic uncertainty on the $v_{\tau}$ flux estimation. The DsTau experiment aims to reduce the systematic uncertainty in the current $v_{\tau}$ cross section measurement to $10 \%$ or below, by measuring the $D_{s}$ differential production cross section (especially longitudinal dependence). For this purpose, emulsion detectors with a spatial resolution of $50 \mathrm{~nm}$ will be used allowing the detection of $D_{s} \rightarrow \tau \rightarrow X$ double kinks in a few mm range. During the physics runs, $2.3 \times 10^{8}$ proton interactions will be collected in the tungsten target, and 1000 $D_{s} \rightarrow \tau$ decays will be detected. In addition to the primary aim, the analysis of $\mathscr{O}\left(10^{5}\right)$ charmed particle pairs can provide valuable by-products such as studies of the forward charm production and the intrinsic charm content in proton. Results from the test runs and the pilot run will be presented together with a prospect for the physics runs in 2021 and 2022.

European Physical Society Conference on High Energy Physics - EPS-HEP2019 -

10-17 July, 2019

Ghent, Belgium

${ }^{*}$ Speaker. 


\section{Study of tau neutrinos}

There are only a few measurements for tau neutrinos and their properties are not well studied. In particular, the $v_{\tau}$ charged-current (CC) cross section is known with much larger statistical and systematical uncertainties compared to the other neutrino flavors, as shown in Figure 1. Moreover, a precise measurement of the $v_{\tau}$ CC cross section would be interesting to test the lepton universality in neutrino scattering. The measurement of the $v_{\tau} \mathrm{CC}$ cross section has also a practical impact on current and future neutrino oscillation experiments. Mass hierarchy measurements in Super-Kamiokande [1] and accelerator neutrino experiments such as DUNE [2] and HyperKamiokande [3] rely on $v_{e}$ flux measurements. The systematic uncertainty from the $v_{\tau}$ interaction cross section could be a limiting factor in their analyses due to $v_{\tau}$ interactions with $\tau \rightarrow e$ decays.

The $v_{\tau}$ interaction cross section was only measured in DONuT [4], OPERA [5] and SK [6] but with large statistical and/or systematical uncertainties of 30-50\% due to low statistics and experimental uncertainties. In a future experiment SHiP [7] at CERN, it is expected to collect thousands of $v_{\tau}$ interactions, providing a negligible statistical uncertainty in the cross section measurement. Thus the overall uncertainty of the cross section will be determined by the systematic uncertainties, especially by the $v_{\tau}$ flux uncertainty, which will be studied by this experiment $[8,9]$. The dominant source of $v_{\tau}$ in the accelerator-based neutrino beam is leptonic decays of $D_{s}^{ \pm}$mesons produced in proton-nucleus interactions. It is necessary to know the differential production cross section of $D_{s}$ to estimate the flux of $v_{\tau}$ 's that pass through the detector. Conventionally, the differential production cross section of charmed particles is approximated by a phenomenological formula $d^{2} \sigma / d x_{F} d p_{T}^{2} \propto\left(1-\left|x_{F}\right|\right)^{n} e^{-b p_{T}^{2}}(1)$, where $x_{F}$ is Feynman $\mathrm{x}$ and $p_{T}$ is transverse momentum. $n$ and $b$ are the parameters controlling the longitudinal and transverse dependence of the differential production cross section, respectively. Although there were several measurements on charm particles as summarized in [10], there is a lack of measurements on the $D_{s}$ differential production cross section in $400 \mathrm{GeV}$ proton interactions, especially concerning the longitudinal dependence represented by the parameter $n$. This has been the main source of the uncertainty of the $v_{\tau}$ cross section measurements. A new measurement of differential production cross section of $D_{s}$ is necessary for precise $v_{\tau}$ measurements in future experiments.
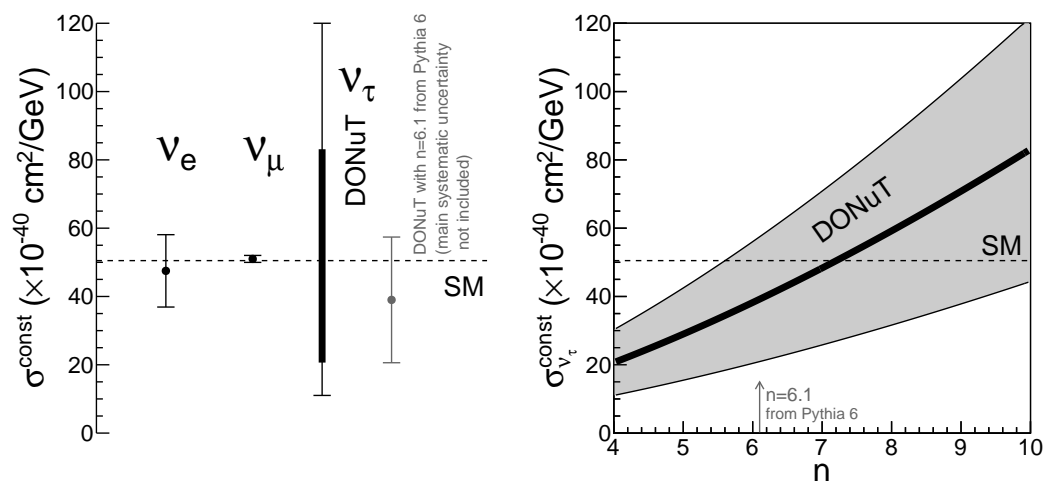

Figure 1: Left: $v, \bar{v}$ averaged cross section of the three neutrino flavors. For the DONuT's result, the value is plotted in the empirical range of the parameter $n$ in formula (1). Right: The cross section result from the DONuT experiment [4]. 


\section{Physics goals}

In the DsTau experiment, a direct measurement of $v_{\tau}$ production will be performed by detecting $D_{s} \rightarrow \tau \rightarrow X$ decays following high-energy proton-nucleus interactions. The project aims to detect $\sim 1000 D_{s} \rightarrow \tau \rightarrow X$ decays in $2.3 \times 10^{8}$ proton interactions with the tungsten target.

DsTau will provide a differential cross section of $D_{s}$ production in $400 \mathrm{GeV}$ proton-nucleus interaction, which can be directly implemented in future experiments. It could be fit with the phenomenological formula (1), and the parameter $n$ could be estimated. The expected precision of the parameter $n$ as a function of the number of detected events is shown in Figure 2. With the statistics of $1000 D_{s} \rightarrow \tau \rightarrow X$ detected events, the relative uncertainty of the $v_{\tau}$ flux will be reduced to below $10 \%$.

In addition to the primary aim of studying $v_{\tau}$ production, a high yield of $\mathscr{O}\left(10^{5}\right)$ charmed particle pairs is expected. The analysis of those events can provide valuable by-products, such as measurements of the forward charm production, the intrinsic charm content in proton [11], the interaction cross section of charmed hadrons.

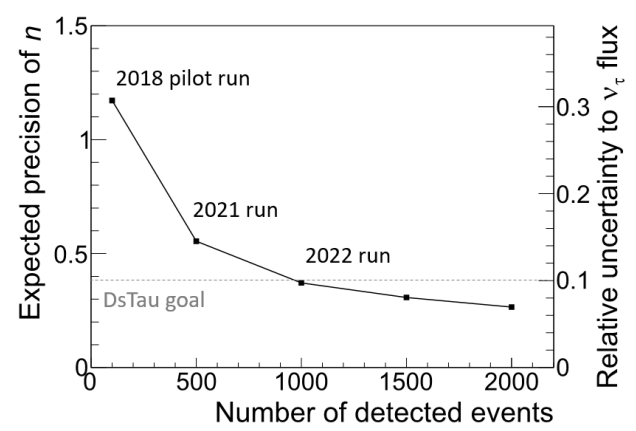

Figure 2: Expected precision for the measurement of parameter $n$ as a function of the number of detected $D_{s} \rightarrow \tau \rightarrow X$ events.

\section{The DsTau detector}

The principle of the experiment is to detect the topology of $D_{s} \rightarrow \tau \rightarrow X$ events appears as a double-kink as shown in Figure 3 (left). In addition, another decay of a charged/neutral charmed particle will be observed with a flight length of a few millimetres. Such a double-kink plus decay topology in a short distance is a very peculiar signature of this process.

The basic unit is made of a 500- $\mu \mathrm{m}$-thick tungsten plate as a target followed by 10 emulsion films interleaved with $200-\mu$ m-thick plastic sheets which act as a decay volume for short-lived particles as well as high-precision particle trackers. A module comprises ten such units followed by the so-called Emulsion Cloud Chamber for momentum measurement of the daughter particles. The whole detector module is $12.5 \mathrm{~cm}$ wide, $10 \mathrm{~cm}$ high and $8.6 \mathrm{~cm}$ thick and consists of a total of 131 emulsion films. During the physics runs, a total of 370 modules will be exposed to the 400 $\mathrm{GeV}$ proton beam. 

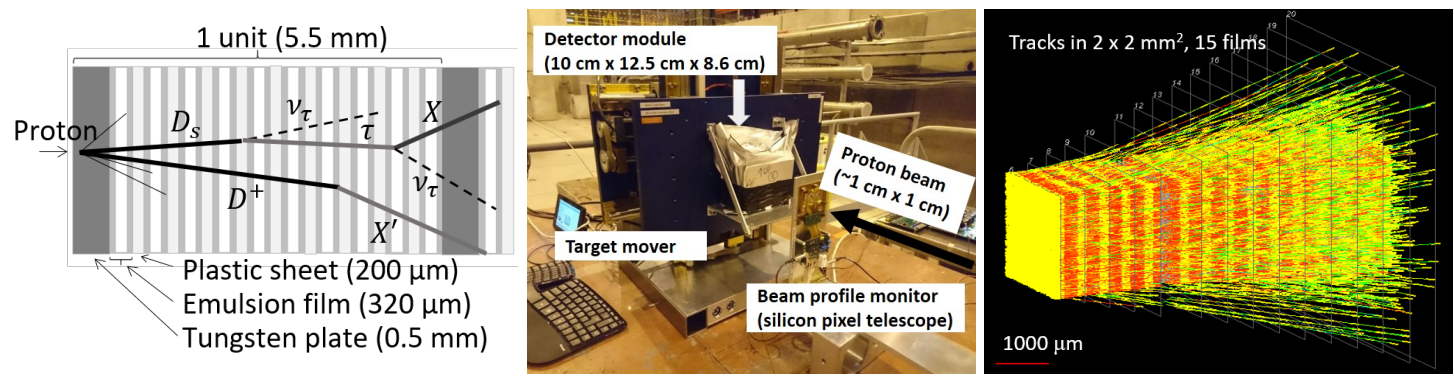

Figure 3: Left: Schematic view of the basic unit. Middle: Detector setup for the test beam at the CERN SPS H4 beamline. The module was driven by a target mover so that it was uniformly exposed to the proton beam. Right: 3D display of the reconstructed tracks.

The detection efficiency for the $D_{s} \rightarrow \tau \rightarrow 1$ prong events is estimated by PYTHIA simulation to be $20 \%$. The momentum of $D_{s}$ will be reconstructed by means of a machine-learning algorithm using topological variables such as flight lengths $\left(F L_{D_{S}}, F L_{\tau}\right)$ and kink angles $\left(\delta \theta_{D_{s} \rightarrow \tau}, \delta \theta_{\tau \rightarrow X}\right)$. The combination of these four variables provides an estimate of $P_{D_{s}}$. The resolution is estimated to be $20 \%$.

\section{Results from the test runs and the pilot run}

Two test runs were conducted at the CERN SPS H2 and H4 beam lines in 2016 and 2017 to test and improve the detector structure and exposure scheme. In 2018, a pilot run was conducted, collecting $10 \%$ of the full statistics collected. Figure 3 (middle) shows the detector setup at the H4 beamline. To have uniform irradiation of the detector, the beam spot was enlarged to roughly 1 $\mathrm{cm} \times 2 \mathrm{~cm}$ by tuning the beam optics.

The event analysis is based on readout of the full emulsion detectors by the Hyper Track Selector (HTS) system [12] with the readout speed of $0.45 \mathrm{~m}^{2} /$ hour/layer, which is the fastest system at present. Readout of emulsion films from the pilot run is in progress and about $86 \%$ of the total will be finished by the end of 2019. The reconstructed tracks (Figure 3 (right)) are then used to find vertices. A systematic search for the decay topology of short-lived particles is applied to the reconstructed vertices. Events with a charged particle decay and another charged or neutral decay are selected. An example of double charm event candidate found by this analysis scheme is shown in Figure 4. The statistics of the found vertices and events with the double decay topology observed in a sub-sample of the data are shown in Table 1. It is consistent with the prediction. The decay length distribution of the data and the FLUKA simulation is shown in Figure 5. Events with decay topologies will be further analyzed by dedicated high-precision systems [13] to detect $D_{s} \rightarrow \tau$ decays.

\section{Summary and prospects}

The DsTau experiment will study $v_{\tau}$ production following high energy proton interactions, will provide essential inputs for future $v_{\tau}$ experiments and pave a way for the search of new physics effects in $v_{\tau}$-nucleon CC interactions. The letter of intent and proposal of DsTau were reviewed by CERN-SPSC. DsTau was then approved by CERN in June 2019 as NA65. 


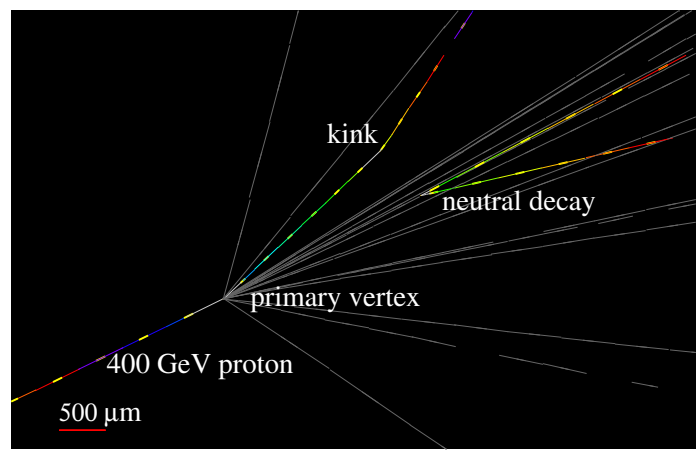

Figure 4: A double charm candidate event with a neutral 2-prong (vee) and a charged 1-prong (kink) topology (tilted view) [10].

\begin{tabular}{|c|c|c|c|}
\hline & Observed & \multicolumn{2}{|c|}{ Expected } \\
\hline Vertices in tungsten & 29297 & \multicolumn{2}{|c|}{$28390 \pm 910$ (syst) } \\
\hline & & Signal & Background \\
\hline Double decay topology & 19 & $13.5 \pm 3.2$ & $2.4 \pm 0.3$ \\
\hline
\end{tabular}

Table 1: Statistics found in the sub-sample of data in which 5629670 protons are analyzed in one unit (1 tungsten plate) [10].
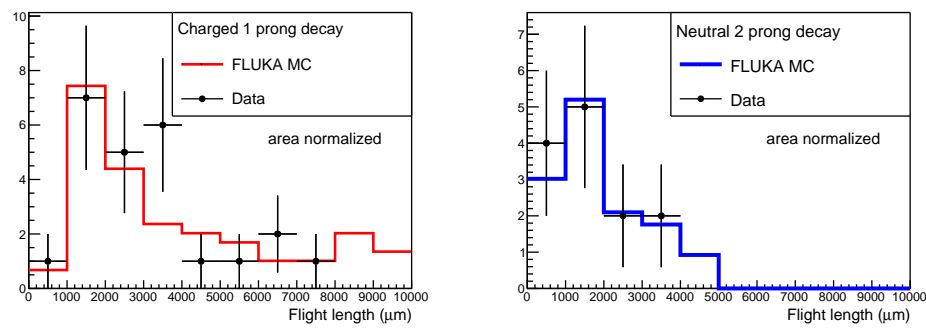

Figure 5: Flight length distributions for charged 1-prong and neutral 2-prong decay candidates in the doublecharm event samples [10].

The test runs in 2016-2017 and the pilot run in August 2018 were performed, in which we accumulated over 20 million proton interactions in the detector. The readout and analysis of these samples are in progress. The results provide a proof of the feasibility of the full-scale physics runs in 2021 and 2022. DsTau will provide a $v_{\tau}$ flux prediction for future experiments with accuracy under $10 \%$. The systematic uncertainty of the $v_{\tau} \mathrm{CC}$ cross section measurement can be sufficiently reduced.

\section{Acknowledgments}

We would thank for the support from the beam physicists at CERN and the SPS coordinator. We acknowledge the technical staffs from LHEP Bern, the colleagues from Nagoya University for sharing the expertise of emulsion detector, and those who helped the beam exposure campaign from Middle East Technical University and from Nagoya University. This work was supported by JSPS KAKENHI Grant Numbers JP 18KK0085 and JP 17H06926. 


\section{References}

[1] Y. Fukudaet al.[Super-Kamiokande Collaboration] Measurement of a small atmospheric $v_{\mu} / v_{e}$ ratio, Phys. Let. B 433 (1998) issue 1, p. 9.

[2] R. Acciarriet al.[DUNE Collaboration], Long-Baseline Neutrino Facility (LBNF) and Deep Underground Neutrino Experiment (DUNE): Volume 1: The LBNF and DUNE Projects, FERMILAB-DESIGN-2016-01 (2016).

[3] K. Abeet al.[Hyper-Kamiokande Proto-Collaboration], Physics potential of a long-baseline neutrino oscillation experiment using a J-PARC neutrino beam and Hyper-Kamiokande,PTEP 2015 (2015) $053 \mathrm{C} 02$.

[4] K. Kodama et al. [DONuT Collaboration], Final tau-neutrino results from the DONuT experiment, Phys. Rev. D 78 (2008) 052002.

[5] N. Agafonova et al. [OPERA Collaboration], Final Results of the OPERA Experiment on $v_{\tau}$ Appearance in the CNGS Neutrino Beam, Phys. Rev. Lett. 120 (2018) no.21, 211801. Erratum: Phys. Rev. Lett. 121 (2018) no.13, 139901.

[6] Z. Li et al. [Super-Kamiokande Collaboration], Measurement of the tau neutrino cross section in atmospheric neutrino oscillations with Super-Kamiokande, Phys. Rev. D 98, 052006 (2018).

[7] M. Anelliet al. [SHiP Collaboration], A facility to Search for Hidden Particles (SHiP) at theCERN SPS, CERN-SPSC-2015-016, SPSC-P-350 (2015).

[8] S. Aoki et al. [DsTau Collaboration], Experimental Proposal, Study of tau-neutrino production at the CERN-SPS, CERN-SPSC-2017-029, SPSC-P-354.

[9] T. Ariga [DsTau Collaboration], Study of tau-neutrino production at the CERN SPS, PoS ICHEP2018 (2019) 240.

[10] S. Aoki et al. [DsTau Collaboration], DsTau: study of tau neutrino production with $400 \mathrm{GeV}$ protons from the CERN-SPS, J. High Energ. Phys. 2020 (2020) 33.

[11] Weidong Bai, Mary Hall Reno, Prompt neutrinos and intrinsic charm at SHiP, arXiv:1807.02746.

[12] M. Yoshimoto, T. Nakano, R. Komatani, H. Kawahara, Hyper-track selector nuclear emulsion readout system aimed at scanning an area of one thousand square meters, PTEP 10 (2017) 103.

[13] A. Ariga and T. Ariga, Fast $4 \pi$ track reconstruction in nuclear emulsion detectors based on GPU technology, JINST 9 (2014) P04002. 